\title{
New Approach to Slowing the Progression of Chronic Kidney Disease
}

\author{
Richard Solomon \\ Nephrology, The University of Vermont Medical Center, Burlington, VT, USA
}

Chronic kidney disease (CKD) affects approximately 37 million people in the USA. Each year, approximately 100,000 progress to end-stage kidney disease (ESKD) and require either dialysis or transplantation. The health care costs are staggering. Medicare spending for CKD is $\sim$ USD 79 billion and $\sim$ USD 35 billion for ESKD. The total costs of USD 114 billion are nearly a quarter of all Medicare expenditures [1].

Much is known about the underlying mechanisms that drive the progression of CKD to ESKD. Two broad pathophysiologic processes have been identified and represent targets for therapy. First, when nephrons are damaged, the remaining undamaged nephrons increase their function in a compensatory manner to maintain homeostasis. This occurs through hemodynamic changes at the level of the vasculature and glomeruli. While beneficial in the short term, there are long-term adverse consequences of these hemodynamic changes that contribute to further loss of nephrons. Second, inflammation drives fibrosis and further loss of nephrons. Inflammation is part of the normal repair process but when sustained, for example by generation of reactive oxygen species, fibrosis is enhanced.

Since the 1980s, we have had multiple prospective randomized trials demonstrating the efficacy of inhibitors of the renin-angiotensin-aldosterone system (RAAS) to delay the progression of CKD by interfering with the hemodynamic mechanisms. These agents now have an established role in the management of patients with CKD. A new class of agents that inhibit the sodium- and glucose-linked transporter proteins (SGLT2 inhibitors) also decrease the progression of CKD in patients with diabetes, again through hemodynamic mechanisms. Importantly, treatment with all these agents slows progression but does not stop it. Loss of kidney function may decrease by $25-50 \%$. However, the rate of loss remains 2- to 4 -fold greater than the rate associated with the aging process.

Reducing inflammation has been a more difficult target. There are many overlapping processes that drive inflammation and ultimately fibrosis and targeting just one of these processes is unlikely to be effective. Recent work using kidney biopsies from patients with a variety of CKD etiologies has looked at the expression of genes that drive fibrosis or mitochondrial dysfunction (a process associated with inflammation through generation of reactive 
oxygen species). These studies identified Nrf2 as a central player in both processes [2]. On the fibrosis side, Nrf2 inhibits NFKB-driven upregulation of profibrotic cytokines such as TNF $\alpha$, IL-6, TGF $\beta$ and IL-1 $\beta$. On the mitochondrial side, Nrf2 stimulates mitochondrial function, leading to increases in ATP and decreases in reactive oxygen species.

Bardoxolone methyl is an Nrf2 agonist that has been studied in randomized trials in patients with CKD from diabetes or Alport's syndrome. It has been found to slow progression in patients already on maximum tolerated doses of RAAS inhibitors. Compared to RAAS or SGLT2 inhibitors, which through their hemodynamic effects tend to reduce GFR during initial therapy, bardoxolone methyl raises GFR and this effect appears to be sustained even after bardoxolone methyl is discontinued. This increase in GFR is not the result of an alteration in hemodynamics but rather is mediated by an increase in filtration surface area. These results are very encouraging. They suggest another effective strategy for delaying the progression of CKD.

In this journal, two papers address the safety of bardoxolone methyl. Hypomagnesemia was reported in $21 \%$ of patients randomized to bardoxolone versus $6 \%$ in the placebo patients in the BEACON trial of 2,185 diabetic patients. The changes in magnesium were reversible after the bardoxolone methyl was stopped. In the first paper involving a substudy of BEACON, intracellular (using sublingual epithelial cells) magnesium was not altered at 28 or 56 days of therapy with 10 or $20 \mathrm{mg}$ of bardoxolone methyl compared to baseline in 24 patients; 24 -h urinary magnesium fell in parallel with the decrease in serum magnesium and fractional magnesium excretion remained unchanged [3]. These findings argue against renal magnesium wasting as a cause of the reduction in serum magnesium. In a second paper involving 149 healthy subjects, there were no significant changes in the QTc interval (QTc Fridericia) following 20 or $80 \mathrm{mg}$ of bardoxolone methyl [4]. This data is further support that the small reductions in serum magnesium are not associated with adverse cardiac effects.

These are encouraging preliminary findings and should support the continued development of this new approach to slowing the progression of CKD. This is an exciting development for the nephrology community and the patients they serve.

\section{References}

1 United States Renal Data System - 2018 Annual Data Report. vol. 1 (CKD), chapt. 7; vol. 2 (ESRD), chapt. 9. Available from: https://www.usrds.org/adrchapters.aspx.

2 Martini S, Nair V, Keller BJ, Eichinger F, Hawkins JJ, Randolph A, et al.; European Renal cDNA Bank; C-PROBE Cohort; CKDGen Consortium. Integrative biology identifies shared transcriptional networks in CKD. J Am Soc Nephrol. 2014 Nov;25(11):2559-72.

3 Rizk DV, Silva AL, Pergola PE, Toto R, Warnock DG, Chin MP, et al. Effects of bardoxolone methyl on magnesium in patients with type 2 diabetes mellitus and chronic kidney disease. Cardiorenal Med. 2019 Jun;1-10.

4 Chin MP, Rich S, Goldsberry A, O Apos Grady M, Meyer CJ. Effects of bardoxolone methyl on QT interval in healthy volunteers. Cardiorenal Med. 2019 Jun;1-8. 